\title{
Protocol for a two-arm pragmatic stepped- wedge hybrid effectiveness- implementation trial evaluating Engagement and Collaborative Management to Proactively Advance Sepsis Survivorship (ENCOMPASS)
}

Marc Kowalkowski ${ }^{1 *}$ D, Tara Eaton ${ }^{1}$, Andrew McWilliams ${ }^{1,2}$, Hazel Tapp ${ }^{3}$, Aleta Rios ${ }^{4}$, Stephanie Murphy², Ryan Burns', Bella Gutnik', Katherine O'Hare ${ }^{3}$, Lewis McCurdy ${ }^{5}$, Michael Dulin ${ }^{6,7}$, Christopher Blanchette ${ }^{7,8}$, Shih-Hsiung Chou', Scott Halpern ${ }^{9,10}$, Derek C. Angus ${ }^{11,12}$ and Stephanie P. Taylor ${ }^{2}$

\begin{abstract}
Background: Sepsis survivors experience high morbidity and mortality, and healthcare systems lack effective strategies to address patient needs after hospital discharge. The Sepsis Transition and Recovery (STAR) program is a navigator-led, telehealth-based multicomponent strategy to provide proactive care coordination and monitoring of high-risk patients using evidence-driven, post-sepsis care tasks. The purpose of this study is to evaluate the effectiveness of STAR to improve outcomes for sepsis patients and to examine contextual factors that influence STAR implementation.
\end{abstract}

Methods: This study uses a hybrid type I effectiveness-implementation design to concurrently test clinical effectiveness and gather implementation data. The effectiveness evaluation is a two-arm, pragmatic, stepped-wedge cluster randomized controlled trial at eight hospitals in North Carolina comparing clinical outcomes between sepsis survivors who receive Usual Care versus care delivered through STAR. Each hospital begins in a Usual Care control phase and transitions to STAR in a randomly assigned sequence (one every 4 months). During months that a hospital is allocated to Usual Care, all eligible patients will receive usual care. Once a hospital transitions to STAR, all eligible patients will receive STAR during their hospitalization and extending through 90 days from discharge. STAR includes centrally located nurse navigators using telephonic counseling and electronic health record-based support to facilitate best-practice post-sepsis care strategies including post-discharge review of medications, evaluation for new impairments or symptoms, monitoring existing comorbidities, and palliative care referral when appropriate. Adults admitted with suspected sepsis, defined by clinical criteria for infection and organ failure, are included. Planned enrollment is 4032 patients during a 36-month period. The primary

\footnotetext{
* Correspondence: Marc.Kowalkowski@AtriumHealth.org

${ }^{1}$ Center for Outcomes Research and Evaluation, Atrium Health, 1300 Scott Ave, Charlotte, NC 28203, USA

Full list of author information is available at the end of the article
}

C C The Author(s). 2021 Open Access This article is licensed under a Creative Commons Attribution 4.0 International License, which permits use, sharing, adaptation, distribution and reproduction in any medium or format, as long as you give appropriate credit to the original author(s) and the source, provide a link to the Creative Commons licence, and indicate if changes were made. The images or other third party material in this article are included in the article's Creative Commons licence, unless indicated otherwise in a credit line to the material. If material is not included in the article's Creative Commons licence and your intended use is not permitted by statutory regulation or exceeds the permitted use, you will need to obtain permission directly from the copyright holder. To view a copy of this licence, visit http://creativecommons.org/licenses/by/4.0/ The Creative Commons Public Domain Dedication waiver (http://creativecommons.org/publicdomain/zero/1.0/) applies to the data made available in this article, unless otherwise stated in a credit line to the data. 
effectiveness outcome is the composite of all-cause hospital readmission or mortality within 90 days of discharge. A mixedmethods implementation evaluation will be conducted before, during, and after STAR implementation.

Discussion: This pragmatic evaluation will test the effectiveness of STAR to reduce combined hospital readmissions and mortality, while identifying key implementation factors. Results will provide practical information to advance understanding of how to integrate post-sepsis management across care settings and facilitate implementation, dissemination, and sustained utilization of best-practice post-sepsis management strategies in other heterogeneous healthcare delivery systems.

Trial registration: NCT04495946. Submitted July 7, 2020; Posted August 3, 2020.

Keywords: Sepsis, Infection, Continuity of patient care, Patient navigator, Health services, Pragmatic clinical trial

\section{Contributions to the literature}

- Sepsis survivors experience high morbidity and mortality, and healthcare systems lack effective strategies to address patient needs after hospital discharge.

- The STAR program extends nurse navigator support during post-sepsis care to facilitate targeted, evidence-driven recommendations using an integrated, patient-centric telehealth approach.

- The proposed study combines a pragmatic randomized trial framework with longitudinal implementation science and prospective economic evaluations to compare usual care versus care delivered with added STAR program support for improving post-sepsis care and outcomes.

- Research will add new practical information to advance understanding of how to best integrate effective post-sepsis management across care settings and heterogeneous healthcare systems.

\section{Background}

Sepsis is a common, life-threatening condition defined by organ dysfunction due to a dysregulated response to infection [1]. Recent policy initiatives have helped to improve timely sepsis identification and treatment and decrease hospital mortality for patients hospitalized with sepsis [2-4]. However, sepsis survivors continue to face challenges after the acute illness episode and experience poor long-term outcomes, including new functional, cognitive, and psychological deficits, and high rates of hospital readmission and mortality in the 90-days after hospital discharge [4-11].

We developed the Sepsis Transition and Recovery (STAR) program to address persistent morbidity and mortality for sepsis survivors. The STAR program uses telehealth nurse navigation to deliver a bundle of bestpractice care elements for longitudinal post-sepsis care up to 90 days. These care elements are directed towards the specific challenges and sequelae following a sepsis hospitalization and include: 1) identification and treatment of new physical, mental, and cognitive deficits; 2) review and adjustment of medications; 3) surveillance of treatable conditions that commonly lead to poor outcomes including chronic conditions that may de-stabilize during sepsis and recovery; and 4) focus on palliative care when appropriate [12]. Prior observational data have shown these care elements to be associated with improved outcomes for sepsis survivors [13]. However, they are not widely applied in real-world settings for this vulnerable population, likely hindered by a gap in understanding key contextual factors underlying how to best integrate this bundle of care elements into the complex and fragmented post-discharge setting [14-19].

The objectives of the Engagement and Collaborative Management to Proactively Advance Sepsis Survivorship (ENCOMPASS) trial are to concurrently evaluate: i) the effectiveness of the STAR intervention for reducing mortality and hospital readmission assessed 90 days after hospitalization among high-risk sepsis survivors, and ii) contextual factors related to the real-world implementation of the program. Furthermore, because implementing support programs such as STAR requires health system investments, we will also evaluate the effect of these investments on costs and cost-effectiveness.

\section{Methods \\ Study design}

ENCOMPASS is an effectiveness-implementation hybrid type I trial. The evaluation component is designed as a two-arm, pragmatic, stepped-wedge cluster randomized controlled trial conducted at eight hospitals in which each participating hospital begins in a usual care control phase and transitions to the STAR program intervention in a randomly assigned sequence, with one of eight hospitals assigned to transition at each four-month interval (i.e., step; Fig. 1). Participant enrollment and randomization will take place from July 2020 through June 2023. During the time that a hospital is allocated to usual care, all eligible patients will receive usual care. Once a hospital has been allocated to the STAR arm, all eligible patients will receive STAR during their index hospitalization and extending through 90 days from discharge or date of death. We will capture patient eligibility and outcomes directly 


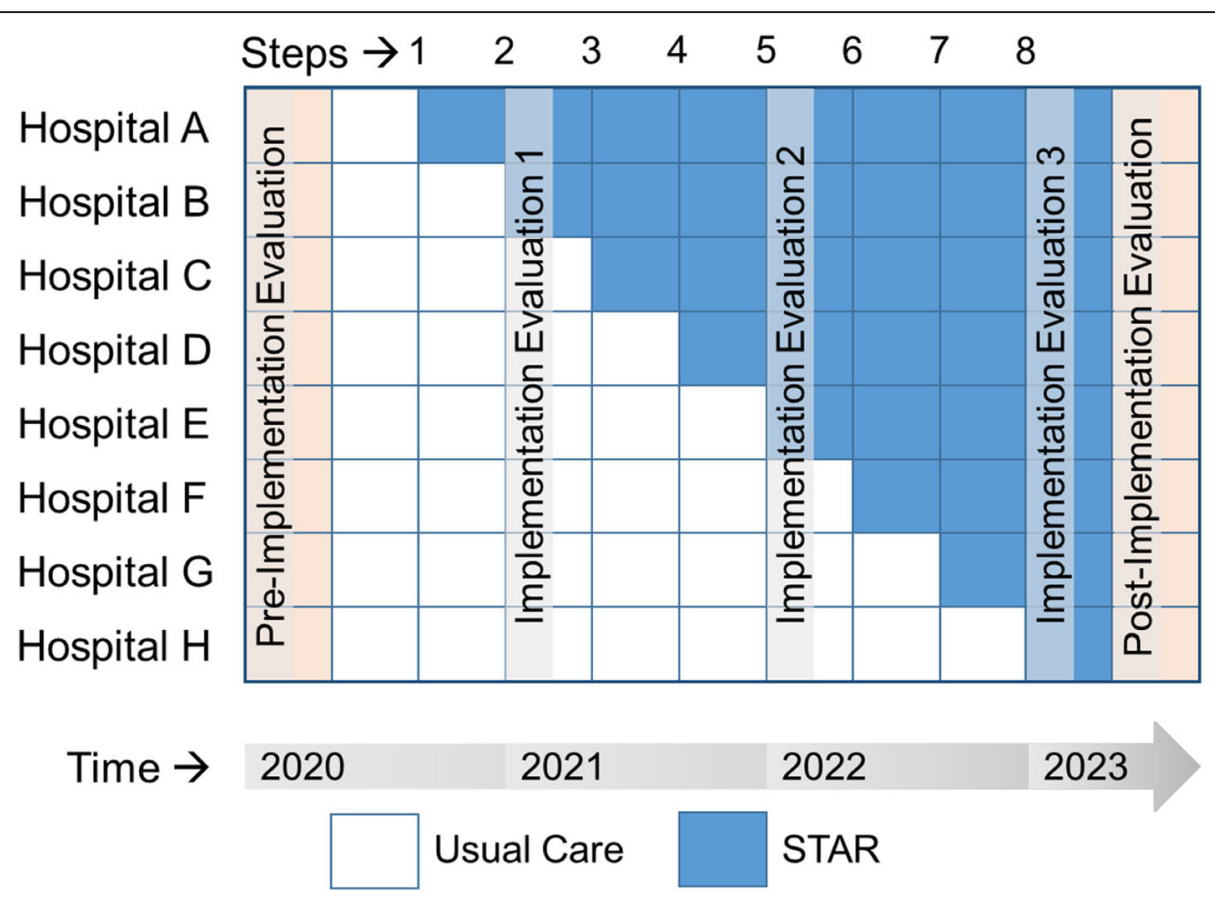

Fig. 1 Overview of ENCOMPASS Stepped-Wedge Trial Design and Timeline. The ENCOMPASS trial design is depicted. Each study hospital begins in the Usual Care condition. Every 4 months, one study hospital transitions from Usual Care to the Sepsis Transition and Recovery (STAR) Program for the remainder of the trial. The sequence and timing of the transition for each hospital is randomly assigned. The total ENCOMPASS trial enrollment interval is 36 months. Implementation is evaluated before, during, and after the patient enrollment interval

from routinely collected data housed in the EHR system and data warehouse. Primary and secondary clinical outcomes will be assessed 90 days after discharge from index hospitalization. We will also use a mixed methods approach to examine implementation of STAR and inform best practices for future dissemination of transition services for sepsis patients.

The hybrid type I trial design combining comparative effectiveness evaluation with collection of implementation outcomes is optimal for ENCOMPASS because STAR satisfies previously described design considerations [20], including 1) there is strong face validity for the recommended post-sepsis care elements and extends existing evidence for delivery of successful care transitions interventions leveraging nurse navigation; 2 ) indirect evidence supports an association between delivery of the care elements and reduced readmission and mortality [13]; 3) the intervention is associated with minimal risk; and 4) there is "implementation momentum" for the adoption of focused transition support services for high-risk patients $[21,22]$. We selected a stepped-wedge randomized design for ENCOMPASS, rather than patient-level randomization, because the intervention is delivered at the hospital level and individual patient randomization could potentiate contamination. The stepped-wedge design also has practical advantages over traditional parallel-cluster randomized designs because it allows for staggered implementation of the intervention across all hospitals, thereby promoting hospital enthusiasm for participating and providing time to prepare each hospital for deployment of the intervention [23, 24]. This trial was approved by the Advarra Institutional Review Board (IRB) with a waiver of informed consent as this evaluation utilizes elements routinely collected in usual clinical practice and deemed to present minimal risk to study participants (IRB\#Pro00036873 v1.0 June 26, 2019). The trial is registered with ClinicalTrials.gov (NCT\#04495946), and the trial protocol adheres to the Standardized Protocol Items: Recommendations for Interventional Trials (SPIRIT) guidelines [25] (Additional File), the CONSORT extension for cluster randomized trials [26], and the Pragmatic Explanatory Continuum Indicator Summary 2 (PRECIS-2) domains for the design of pragmatic studies [27].

\section{Conceptual framework}

We developed the STAR program to enhance the transition process for sepsis survivors based on the widelyadopted Chronic Care Model (Fig. 2) [28, 29]. The aim of the Chronic Care Model is to transform patient care from acute and reactive to proactive and planned via six interrelated system changes intended to promote productive interactions between an informed, activated patient and prepared, proactive care team. In STAR, nurse 


\section{Community \\ Resources \& Policies}

STAR: Appropriate use of community partners (e.g., social

services, paramedicine, community healthcare providers)

Health System

Organization

Self-Management Support

STAR: synchronous communication, disease-specific education, stoplight chart for self monitoring, proactive engagement, planning, medication management, shared decision making around goals of care through motivational interviewing, psychosocial support
Delivery System Support

STAR: regular contacts with longitudinal follow-up, care coordination, centralized networked connectivity and support, established links to clinical teams, clearly defined roles of navigator and care team

Decision
Support
STAR: data-
driven
algorithms for
targeted patient
identification,
best-practice
guidelines,
robust
operational
processes,
protocols for
escalation of
care

Information Support

STAR: electronic health record and care management system, secure messaging service, telephone and telehealth strategies

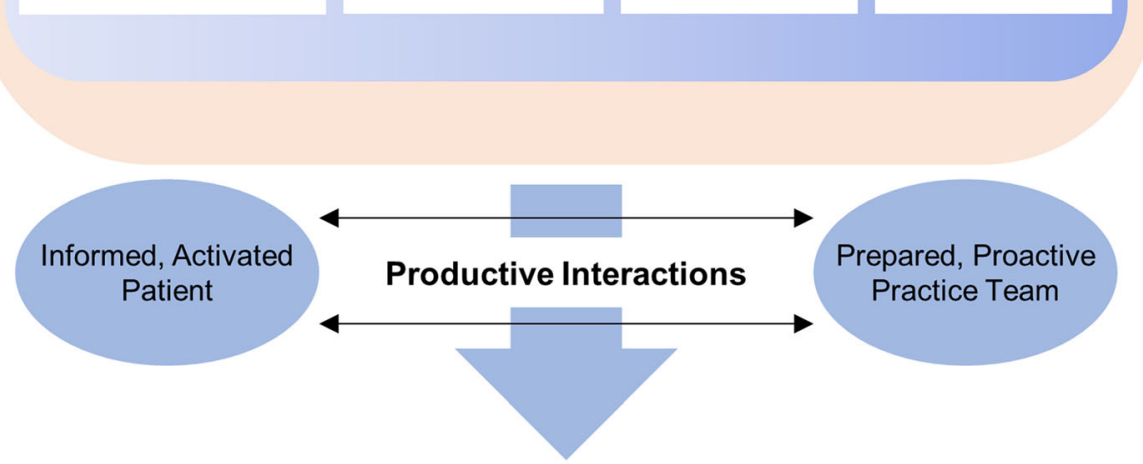

\section{Improved Health Outcomes}

Fig. 2 Conceptual model describing the integration of STAR to improve post-sepsis care and outcomes. The elements of the Sepsis Transition and Recovery (STAR) program are shown mapped onto the Chronic Care Model framework. Adapted from Wagner EH, Chronic disease management: what will it take to improve care for chronic illness? Eff Clin Pract. 1998

navigators are trained to provide disease and health system education, help patients overcome medical system barriers to recommended care, and bridge gaps in service that can serve as points of failure for complex sepsis patients [30]. Additionally, navigators function to address insurance and other financial barriers, aid in care coordination, increase appropriate referrals to community resources, and use motivational interviewing to encourage behavior change, increase proactive patientprovider interactions and improve outcomes [31-34].

The implementation determinants evaluation in ENCOMPASS is conceptually guided by the Consolidated Framework for Implementation Research (CFIR) [35, 36]. We will use the five CFIR domains (intervention characteristics, outer setting, inner setting, characteristics of individuals, and process) to guide planning, organization, and conduct of STAR implementation and to evaluate and adapt the implementation strategy, observe fidelity, and examine sustainability and potential for wider dissemination. The breadth of constructs included within the CFIR enables our study to inform how health systems effectively implement interventions that improve outcomes for sepsis survivors across heterogenous settings and diverse populations.

\section{Study setting}

We will test the STAR program intervention within the course of providing usual care among a large and diverse population of post-sepsis patients admitted to eight hospitals within Atrium Health $(\mathrm{AH})$, one of the largest, 
vertically integrated health systems in the US. The eight acute care hospitals participating in this study use the same EHR, which connects across all points of care, including outpatient practices, urgent care locations, emergency departments and hospitals. Additionally, study hospitals vary in size and provide care to diverse regions of North Carolina, representing seven different counties (Table 1). We deliberately selected non-tertiary, geographically diverse acute care hospitals for our study to demonstrate the scalability and generalizability of STAR. Specifically, over one-half of sepsis patients in the US receive care at hospitals that have fewer than 500 beds [37, 38]. Our selection of comparably sized hospitals reflects the importance of broadly disseminating successful implementation strategies to non-tertiary facilities providing sepsis care. The diversity of clinical care, established telehealth capabilities, and integrated EHR platform make Atrium Health an ideal environment for this pragmatic trial to evaluate STAR program implementation across geographically, culturally, and economically diverse settings.

\section{Identification and recruitment of eligible sepsis patients} Prior to the initiation of recruitment for ENCOMPASS, the study team worked with key stakeholders to define sepsis patients with high post-sepsis care needs likely to benefit from the STAR program, based on clinical sepsis criteria and coexisting risk profiles [39]. Consistent with our pragmatic study design concept, eligibility criteria are broad, the sample size is large and diverse, and study procedures are embedded into the context of routine care. Patients are eligible if they present to the emergency department and are subsequently admitted under inpatient or observation status to a participating hospital and meet the following criteria: 1 ) $\geq 18$ years of age upon admission; 2) clinically suspected infection (i.e., antibiotic and bacterial culture orders and two or more markers of systemic inflammatory response syndrome [40, 41] within 24 h of presentation); 3) organ dysfunction, defined as two or more points on either admission Sequential Organ Failure Assessment (SOFA) or quick-
SOFA scores [42-45]; 4) deemed to be at high risk of hospital readmission within 90 days, defined as a readmission risk probability $\geq 25 \%$ using a previously validated model described below; and 5) not discharged from the hospital at the time the daily list of eligible patients is generated each weekday morning.

We will exclude patients: 1 ) with a change in code status (i.e., initially full code followed by change to do not resuscitate and/or do not intubate) within $24 \mathrm{~h}$ after index presentation due to presumed limitation of aggressive treatment and exposure to STAR program components; 2) reside $>2.5 \mathrm{~h}$ drive time from the treating hospital due to the maximum reach of the community services leveraged within the STAR program and the general assumption that these patients may have less comprehensive utilization tracking within $\mathrm{AH}$ record systems for study outcomes; 3 ) are actively participating in a different $\mathrm{AH}$ care management program (e.g., cancer care patient navigation) documented in the EHR at time of hospital admission; or 4) have been previously randomized as part of this study.

Our readmission risk model includes clinical and administrative data selected based on their association with post-sepsis outcomes and conceptual models of postsepsis pathophysiology such as: physiologic measurements, lab values, basic sociodemographic characteristics, and personal medical history [46-48]. All model covariates are sourced from a patient's routinelycaptured clinical data during hospitalization and billing history at the time of hospital admission to produce a near-real time risk score that identifies cohorts at high risk for 90-day hospital readmission. The EHR-based algorithm will run each morning and automatically generate a list of newly eligible, high-risk patients admitted over the prior $72 \mathrm{~h}$. Because we will use a sensitive "suspected sepsis" definition to enroll eligible high-risk patients in near real time, we validated our algorithm against an ultimate determination of sepsis at hospital discharge prior to ENCOMPASS trial recruitment. Our blinded review of 100 consecutively identified patients across the eight participating hospitals found that 55

Table 1 Characteristics of ENCOMPASS participant hospitals

\begin{tabular}{llll}
\hline Hospital & Location & Setting & IP / ICU Beds \\
\hline CHS Blue Ridge & Morganton, NC & Rural & $269 / 16$ \\
AH Cleveland & Shelby, NC & Rural & $241 / 18$ \\
AH Pineville & Charlotte, NC & Urban, community & $235 / 30$ \\
AH Union & Monroe, NC & Suburban, community & $175 / 14$ \\
AH Stanly & Albemarle, NC & Rural & $109 / 10$ \\
AH Lincoln & Lincolnton, NC & Rural & $101 / 10$ \\
AH University City & Charlotte, NC & Urban, community & $100 / 8$ \\
AH Kings Mountain & Kings Mountain, NC & Rural & $67 / 6$ \\
\hline
\end{tabular}

CHS Carolinas Healthcare System, AH Atrium Health, IP Inpatient, ICU Intensive Care Unit 
patients were deemed to have sepsis (i.e., infection with organ dysfunction) at discharge. Using chart review as the reference standard, our EHR algorithm had 93\% sensitivity and $80 \%$ specificity.

\section{Study processes}

For patients in both arms, data from the automated, daily patient list are sent electronically to the study database. During the intervention phase at each facility (i.e., after transition from usual care), the navigator receives the list of admitted, eligible high-risk patients via secure email. The navigator contacts eligible patients at intervention hospitals by telephone and introduces the STAR process before discharge. Our team has successfully deployed these same processes during prior transitions interventions [49]. In this real-world implementation study, patients, clinicians, and investigators are not blinded to group assignments; but, primary outcomes are collected from objective data, minimizing bias introduced by the lack of blinding. At any point, patients may decline participation in STAR or any components of usual care.

\section{Trial interventions}

Arm 1: Usual Care. When assigned to Usual Care, hospitals and their providers will not have access to the STAR program. Patients in the Usual Care group continue to receive routine clinical care throughout their hospitalization and following hospital discharge. Usual Care elements are not prescribed but, in everyday practice, typically consist of: patient education and follow-up instructions at discharge; routine recommendations for timely outpatient follow-up visits; arrangements for home health services, use of existing transitional care services for some patients (e.g., comprehensive discharge planning, health coaching support) [22], or care management follow-up based on each patient's needs but not specifically tailored to the sepsis population; and when necessary receipt of ongoing care in post-acute skilled nursing facility or acute rehabilitation settings but with no sepsis-specific follow-up. To best align our pragmatic trial design with real-world conditions, aspects of Usual Care are determined by treating clinicians independent of trial assignment; however, we will actively surveil and document concurrent system-level interventions that may occur during the study period.

Arm 2: Nurse Navigator-Driven STAR. Patients admitted to hospitals in the intervention arm at time of eligibility determination receive care via the STAR program (Fig. 3). The STAR program is described in detail elsewhere [49]. Broadly, STAR applies the Chronic Care Model organizational framework [28], which promotes care planning, active follow-up, and patient, provider, and community engagement, to increase adherence to best-practice recommendations and improve care coordination between hospital and post-acute care transitions during sepsis recovery. The STAR program employs a centrally-located nurse navigator who has clinical knowledge of sepsis and its cognitive and functional sequelae, core competencies in navigating transitions of care (e.g., facilitating communication, coordinating care, assessing/ addressing barriers to care, providing patient education and practical resource information/referrals), and works as an extension of AH's Transition Services within the Division of Hospital Medicine, which is a multidisciplinary team providing acute care support during the peri-discharge interval [50]. The STAR navigator will provide proactive coordination and monitoring to patients using targeted, evidence-based best-practice care

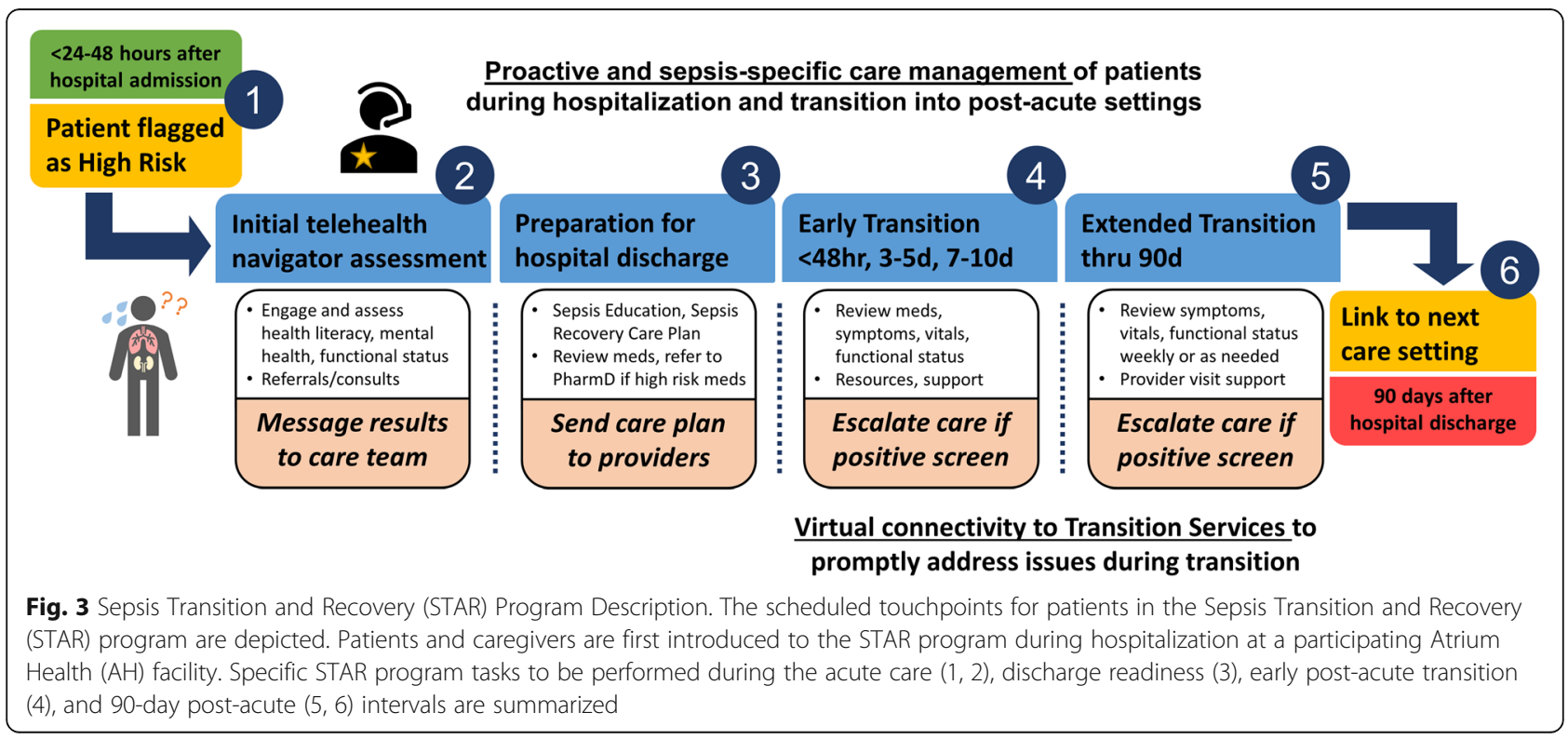


components: i) identification and treatment referral for new physical, mental, and cognitive deficits; ii) review and recommendation for adjustment of medications; iii) surveillance for treatable conditions that commonly lead to poor outcomes; and iv) referral to palliative care when appropriate (Table 2). In the current trial, the STAR navigator will provide telephone- and EHR-based support within the hospitalization and to patients across all discharge settings with remote follow-up at specified intervals throughout the 90 days post hospital discharge. Clinical oversight and care escalation (e.g., facilitation of community paramedicine in-home assessment, telemedicine video consult, in-person clinic visit) is supported by the Transition Services team and complementary to the patient's primary care provider.

\section{Data collection}

All clinical and outcomes data are collected directly from the AH EHR system and Enterprise Data Warehouse
(EDW). Data are collected during the index hospitalization and include: basic sociodemographic characteristics (e.g., age, gender, race/ethnicity, marital status, insurance status at time of enrollment), past medical history (e.g., comorbidities, prior healthcare use), infection and disease severity (e.g., physiologic measurements [e.g., mean arterial pressure], lab values [e.g., complete blood count, basic/comprehensive metabolic panel, lactate]), hospital procedures (e.g., mechanical ventilation), and organizational variables (e.g., hospital location). Additional data are collected on care delivered during follow up and include: physical, speech, and occupational therapy, mental health assessment and referrals to behavioral health, outpatient follow-up visits, documented medication reconciliation in the EHR, Care Alignment Tool completion, and palliative care consultation. Navigators also document completion of STAR workflow processes in the patient's EHR through the care management electronic documentation form, then data is exported into a research database (REDCap) [51].

Table 2 Post-Sepsis Guidelines with Sepsis Transition and Recovery (STAR) Program Task

\begin{tabular}{|c|c|c|}
\hline Core component / Evidence & Recommendation & STAR Task \\
\hline \multicolumn{3}{|c|}{ Screen for new physical, mental, and cognitive deficits after sepsis } \\
\hline $\begin{array}{l}\text { Functional disability: Patients aged } \geq 65 \text { years develop } 1 \\
\text { to } 2 \text { new functional limitations }\end{array}$ & $\begin{array}{l}\text {-Prescribe structured exercise program } \\
\text {-Referral to Physical/ Cardiac/ Pulmonary rehab as } \\
\text { needed }\end{array}$ & $\begin{array}{l}\text { Confirm functional assessment } \\
\text { (Physical Therapy). Refer as } \\
\text { needed. }\end{array}$ \\
\hline $\begin{array}{l}\text { Swallowing impairment: Of patients aged } \geq 65 \text { years, } \\
1.8 \% \text { readmitted }<90 \text { days for aspiration pneumonitis }\end{array}$ & $\begin{array}{l}\text {-Screen for cough, dysphagia, weak voice } \\
\text {-Referral to speech therapy as needed }\end{array}$ & $\begin{array}{l}\text { Confirm screen and team aware. } \\
\text { Refer as needed. }\end{array}$ \\
\hline $\begin{array}{l}\text { Mental Health impairment: Prevalence for clinically } \\
\text { significant anxiety 32\%, depression 29\%, and PTSD 44\% }\end{array}$ & $\begin{array}{l}\text {-Review details of hospital course (e.g., ICU diary) } \\
\text {-Depression screen } \\
\text {-Referral to peer support or Behavioral Health as } \\
\text { needed }\end{array}$ & $\begin{array}{l}\text { Mental health screen. Refer as } \\
\text { needed. }\end{array}$ \\
\hline \multicolumn{3}{|l|}{ Review and Adjust Long-term Medications } \\
\hline $\begin{array}{l}\text { Medication errors: Errors of omission and commission } \\
\text { occur in up to } 25 \% \text { of patients, depending on } \\
\text { medication }\end{array}$ & $\begin{array}{l}\text {-Review antibiotic choice, dose, duration. } \\
\text {-Start/continue meds for comorbidities; adjust for } \\
\text { BMI, etc. } \\
\text {-Discontinue hospital meds without ongoing } \\
\text { indication }\end{array}$ & $\begin{array}{l}\text { Antibiotic Stewardship Medication } \\
\text { Reconciliation Vitals/Weight }\end{array}$ \\
\hline $\begin{array}{l}\text { Anticipate and Mitigate risk for Common and } \\
\text { Preventable Causes of Health Deterioration }\end{array}$ & & $\begin{array}{l}\text { Routine virtual follow up. } \\
\text { Schedule provider visits }\end{array}$ \\
\hline $\begin{array}{l}\text { Infection: Of patients aged } \geq 65 \text { years, } 11.9 \% \text { readmitted } \\
<90 \text { days for infection }(6.4 \% \text { for sepsis })\end{array}$ & $\begin{array}{l}\text {-Patient education about symptoms of sepsis, } \\
\text { recurrence } \\
\text {-Appropriate vaccination } \\
\text {-Monitor for symptomatic improvement in index } \\
\text { infection }\end{array}$ & $\begin{array}{l}\text { Education } \\
\text { Medication Reconciliation Monitor } \\
\text { symptoms }\end{array}$ \\
\hline $\begin{array}{l}\text { Heart failure exacerbation: Of patients aged } \geq 65 \text { years, } \\
5.5 \% \text { readmitted }<90 \text { days for CHF }\end{array}$ & $\begin{array}{l}\text {-Reassess beta-blocker, diuretic, ACE-inhibitor dosing } \\
\text {-Monitor volume status (fluid balance) - recognizing } \\
\text { dry weight may be decreased if muscle mass lost }\end{array}$ & $\begin{array}{l}\text { Medication Reconciliation Vitals/ } \\
\text { Weight } \\
\text { Monitor symptoms }\end{array}$ \\
\hline $\begin{array}{l}\text { Acute Renal Failure: Of patients aged } \geq 65 \text { years, } 3.3 \% \\
\text { readmitted }<90 \text { days for acute renal failure }\end{array}$ & $\begin{array}{l}\text {-Monitor renal function; lab testing as needed } \\
\text {-Reassess need and dosages for renally cleared, } \\
\text { nephrotoxic agents }\end{array}$ & $\begin{array}{l}\text { Monitor symptoms } \\
\text { Confirm CBC/BMP } \\
\text { Medication Reconciliation }\end{array}$ \\
\hline $\begin{array}{l}\text { COPD exacerbation: Of patients aged } \geq 65 \text { years, } 1.9 \% \\
\text { readmitted }<90 \text { days for COPD exacerbation }\end{array}$ & $\begin{array}{l}\text {-Confirm/initiate appropriate controller inhalers } \\
\text {-Appropriate vaccination } \\
\text {-Review use of benzodiazepines/opioids }\end{array}$ & $\begin{array}{l}\text { Monitor symptoms } \\
\text { Medication Reconciliation }\end{array}$ \\
\hline Assess appropriateness for palliative care & $\begin{array}{l}\text {-Palliative Care screen/consult as indicated } \\
\text {-Goals of care. Educate on disease progression/ } \\
\text { terminal }\end{array}$ & $\begin{array}{l}\text { Discuss Palliative Care consult. } \\
\text { Goals of Care }\end{array}$ \\
\hline
\end{tabular}




\section{Study outcomes}

The primary outcome is a dichotomous, composite measure of mortality and hospital readmission assessed 90 days post index hospital discharge. This combined outcome is ideally suited to our pragmatic study design because mortality and hospital readmission are widely regarded as patient-important outcomes [52]. In addition, patients' risks for both mortality and hospital readmission remain elevated in the period following sepsis hospitalization [11,53] and are uniformly captured from data contained in the AH EDW, minimizing nondifferential assessment, outcome misclassification, and missing data. Moreover, preventing avoidable hospital readmission represents a critical quality metric for hospitals and has been targeted as a high priority for health care reform in the United States [54]. Finally, recent evidence from focused readmission reduction initiatives have shown decreased readmission rates [55] but also increased mortality during the same interval [56], highlighting the importance of measuring mortality and readmission rates in combination.

In ENCOMPASS, mortality is defined as any date of death documented in the AH EDW within 90 days of index hospital discharge, including events from national death record data uploaded monthly into the EDW via our institutional subscription [57]. Rehospitalization is defined as any inpatient or observation encounter to any of the more than $40 \mathrm{AH}$ hospitals within the 90 days following index hospital discharge. Both inpatient and observation status rehospitalizations count towards a readmission because either status represents an adverse event important to patients and healthcare systems. In our primary analysis, mortality and hospital readmission events are weighted equally, and patients who experience either mortality or hospital readmission outcomes within 90 days of index hospital discharge are defined as event positive.

The following secondary clinical outcomes are assessed at 90 days after hospital discharge: 1) number of days alive and outside the hospital (i.e., a patientcentered, continuous measure of mortality and hospital readmission days that ranges from zero [most severe outcome] to 90 days [least severe outcome]) [58-60]; 2) all-cause mortality; 3) all-cause hospital readmission; 4) cause-specific hospital readmission with primary diagnoses (based on International Classification of Diseases, 10th revision diagnosis codes) related to: a) sepsis or common infection (i.e., sepsis [61], pneumonia [J13-18], urinary tract infection [N30, N34, N39.0], skin and soft tissue infection [L00-08]), b) chronic lung disease [J4047], c) heart failure [I50], d) acute renal failure [N17], and e) the composite of ambulatory care sensitive conditions (as defined by the Centers for Medicare and Medicaid Services) [62]; 5) number of ED visits; and 6) number of outpatient visits.

\section{Primary statistical analysis}

We will use an intent-to-treat approach to primary and secondary analyses, such that all patients meeting identical criteria and randomized will be analyzed, regardless of adherence to intervention assignment. This real-world approach will assess intervention effectiveness while limiting selection biases associated with adherence. Because hospitals are randomized to STAR in a staggered sequence and the outcome varies at the patient level, we will use a generalized linear mixed-effects model to compare the composite 90-day mortality and hospital readmission primary outcome measure between the intervention conditions [63]. The intervention fixedeffect coefficient will compare STAR versus Usual Care conditions (i.e., Usual Care as reference). We will include fixed effects for hospital cluster and time (i.e., month of enrollment) to adjust for hierarchical data structures and potential confounding due to secular trends and increased STAR program enrollment over time $[63,64]$. Due to the stepped wedge design, there is potential for imbalance in patient- and hospital-level characteristics between study arms. We will include prespecified covariates for patient characteristics at time of enrollment (e.g., age, sex, race, comorbidity score, disease severity) and organizational factors (e.g., hospital location) that may be related to mortality and hospital readmission in our adjusted model for primary analysis. Further, we will test for potential modification of the STAR treatment effect by conducting analyses stratified by pre-specified patient characteristics (e.g., age, comorbidity score, disease severity at trial enrollment) [65-67]. We will also extend our model framework to include interaction terms to assess: 1) treatment effect differences across hospitals; and 2) time effect differences across hospitals. In addition to primary analyses, we will conduct modified intent-to-treat analyses excluding sepsis patients 1) who do not survive index hospitalization since STAR is designed to support patients during their transition out of the hospital; and 2) discharged against medical advice since providers do not have the opportunity to deliver full care and prepare the patient for discharge [68]. Based on published data and internal estimates, we expect less than $6 \%$ of study patients will die during index hospitalization and less than $2 \%$ will be discharged against medical advice. We will present group comparisons as odds ratios and 95\% confidence intervals. Similar to our primary analysis approach, we will construct generalized linear mixed-effects models for individual assessments of secondary outcomes. We will test different distribution parameters to determine the optimal distribution family for each model and outcome variable (e.g., binomial, negative binomial, gamma distributions). All hypothesis tests will be two sided and data will be analyzed using SAS (Cary, NC) or R (Vienna, Austria). 
Methods to Handle Missing Data. We do not anticipate substantial missing data because all outcomes are routinely collected variables and utilization is broadly captured within our large integrated system. Values for patients who do not have health care utilization or mortality records during the study follow-up interval are assumed to be null. While utilization may occur outside $\mathrm{AH}$, this is not expected to be a major limitation because of $\mathrm{AH}$ market share and accessibility. Specifically, $\mathrm{AH}$ operates three large hospitals in Cabarrus and Mecklenburg Counties, the only acute care hospitals in Anson, Burke, Cleveland, Lincoln, Stanly, and Union counties, where most of this study will be conducted, and more than 40 hospitals in the region overall. Additionally, any utilization occurring outside the system is anticipated to be non-differentially distributed between groups and thus impact treatment groups equally. Further, internal historical data indicates nearly $75 \%$ of high-risk patients are Medicare-insured (i.e., Medicare Shared Savings Plan beneficiaries). For these patients, we will have complete healthcare claims within and outside $\mathrm{AH}$ facilities during the study interval, as captured through participation in the local AH-managed Accountable Care Organization. We will conduct subgroup analyses within this Medicare-insured population and will use this data to explore missing data patterns that can be adjusted using pattern-mixture methods in sensitivity analyses $[69,70]$.

\section{Sample size and statistical power}

Based on our internal data from sepsis admissions to the study hospitals between 2015 and 2017, we estimate a total of 4480 patients will be eligible for participation during the 36-month ENCOMPASS trial enrollment period (i.e., approximately 16 high risk patients per hospital per month). From this historical data, we observed a 90-day mortality rate of $18 \%$ and 90 -day hospital readmission rate of $42 \%$. From published literature, a small percentage of deaths $(\sim 1-2 \%)$ and between 22 and $42 \%$ of hospital readmissions after sepsis are preventable [10, 71, 72]. Using a stepped-wedge design $[64,73]$ with eight hospitals and intra-cluster correlation coefficients $\rho=0.1$ to account for the hierarchical data structure and potential clustering of intervention effects within hospitals, nine time periods (including baseline), eight steps with one hospital switching from UC to STAR at each step, and total sample size of at least 4032 patients enrolled over 36 months (i.e., at least $90 \%$ of historical patient volumes), we will have $90 \%$ power $(\alpha=0.05)$ to detect a $8 \%$ absolute change in the composite 90-day mortality and hospital readmission outcome that is important to patients and clinically meaningful.

\section{Implementation evaluation}

Outcome measures for evaluation of the STAR program implementation are summarized in Table 3. We will use baseline assessments of implementation climate and ongoing evaluation of STAR implementation to inform and adapt its execution. Mixed-methods data will be collected through surveys, qualitative semi-structured interviews, and focused ethnography. We will use the CFIR to guide development of data collection, coding, and data analysis [35, 36]. The CFIR provides a standardized taxonomy of operationally defined constructs culled from multiple disciplines relevant to implementation of complex programs. We will apply five CFIR domains: intervention characteristics (e.g., evidence strength and quality, relative advantage, adaptability, trialability, complexity); outer setting (e.g., needs and resources of sepsis patients, cosmopolitanism, healthcare system policies and incentives); inner setting (e.g., hospital and healthcare system networks/communication, hospital and healthcare system implementation climate, readiness); characteristics of individuals (e.g., knowledge and beliefs); and process used to implement the program (e.g., quality and extent of planning, engagement of key stakeholders, evaluation). We will also include elements from the Reach, Effectiveness, Adoption, Implementation, and Maintenance (RE-AIM) [74] model to quantify the reach, effectiveness, and costs of the STAR program and its potential for dissemination and scalability.

We will collect data before, during, and after implementation. For pre- and post-implementation data collection, we will conduct qualitative interviews with eight administrative leaders and eight providers regarding organizational support, culture, and recommendations for STAR implementation and then post-implementation to plan future dissemination. During the implementation phase, we will gather data at three time points (i.e., 8-, 20-, and 32months) from: a) qualitative interviews and surveys with navigators and providers to assess and understand barriers to and enablers of STAR implementation and to help improve access to sepsis care, b) qualitative interviews and surveys with patients and caregivers (recruited from intervention arms) to learn about their experiences with STAR during the peri-discharge transition, opinions and perspectives on services received and not received, and preferences for care, and c) focused ethnography of the navigators to obtain a holistic and nuanced understanding of the navigator's role in the STAR intervention [75, 76]. Interview recordings and ethnographic observation fieldnotes will be transcribed for coding and qualitative analysis using ATLA S.ti v8.0. We will use both inductive and deductive coding strategies guided by the CFIR and constant comparison to perform content analysis, applying iterative comparison of newly coded text with previously coded text of the same theme until final thematic refinement is achieved [77]. We 
Table 3 Summary of Outcome Measures for Evaluation of STAR Program Implementation

\begin{tabular}{|c|c|c|c|c|c|c|c|}
\hline & \multirow[b]{2}{*}{ Assessment } & \multirow[b]{2}{*}{ Evaluation tool } & \multicolumn{5}{|c|}{$\begin{array}{l}\text { Implementation time } \\
\text { point }\end{array}$} \\
\hline & & & Pre & $\begin{array}{l}8 \\
\mathrm{~m}\end{array}$ & $\begin{array}{l}20 \\
m\end{array}$ & $\begin{array}{l}32 \\
\mathrm{~m}\end{array}$ & Post \\
\hline \multicolumn{8}{|l|}{ CFIR Construct Assessed } \\
\hline \multirow[t]{2}{*}{$\begin{array}{l}\text { Outer setting (Patient Needs and resources, Cosmopolitanism, } \\
\text { external policies/incentives) }\end{array}$} & $\begin{array}{l}\text { Administrative } \\
\text { leaders }\end{array}$ & CFIR interviews & $x$ & & & & $x$ \\
\hline & Providers & CFIR interviews & $x$ & & & & $x$ \\
\hline \multirow[t]{3}{*}{$\begin{array}{l}\text { Inner Setting (networks/communication, implementation climate, } \\
\text { readiness) }\end{array}$} & $\begin{array}{l}\text { Administrative } \\
\text { leaders }\end{array}$ & CFIR interviews & $x$ & & & & $x$ \\
\hline & Providers & CFIR interviews & $x$ & $x$ & $x$ & $x$ & $x$ \\
\hline & Navigators & CFIR interviews & & $x$ & $x$ & $x$ & \\
\hline \multirow{3}{*}{$\begin{array}{l}\text { Intervention (evidence strength/quality, relative advantage, } \\
\text { adaptability, trialability, complexity) }\end{array}$} & Administration & CFIR Interviews & & $x$ & $x$ & $x$ & \\
\hline & Providers & CFIR Interviews & & $x$ & $x$ & $x$ & \\
\hline & Navigators & CFIR Interviews & & $x$ & $x$ & $x$ & \\
\hline \multirow[t]{3}{*}{ Individuals (knowledge and beliefs about intervention, self-efficacy) } & Providers & $\begin{array}{l}\text { Knowledge survey } \\
\text { Self-Efficacy survey }\end{array}$ & & $x$ & $x$ & $x$ & \\
\hline & Navigators & $\begin{array}{l}\text { Knowledge survey } \\
\text { Self-Efficacy survey }\end{array}$ & & $x$ & $x$ & $x$ & \\
\hline & $\begin{array}{l}\text { Patients and } \\
\text { Caregivers }\end{array}$ & $\begin{array}{l}\text { Knowledge survey } \\
\text { Self-Efficacy survey }\end{array}$ & & $x$ & $x$ & $x$ & \\
\hline Process (execution) & Navigators & Focused ethnography & & $x$ & $x$ & $x$ & \\
\hline \multicolumn{8}{|l|}{ Other outcomes assessed } \\
\hline \multirow[t]{2}{*}{ Reach, Adoption, and Maintenance } & Patients (Reach) & $\begin{array}{l}\text { 1) Navigator use per } \\
\text { eligible patients } \\
\text { 2) risk prediction for those } \\
\text { enrolled }\end{array}$ & & $x$ & $x$ & $x$ & $x$ \\
\hline & $\begin{array}{l}\text { Providers } \\
\text { (Adoption) }\end{array}$ & $\begin{array}{l}\text { \# of providers with pts. } \\
\text { enrolled }\end{array}$ & & $x$ & $x$ & $x$ & $x$ \\
\hline Effectiveness & $\begin{array}{l}\text { Healthcare } \\
\text { utilization }\end{array}$ & $\begin{array}{l}\text { 90-day mortality or } \\
\text { readmission }\end{array}$ & $x$ & & & & $x$ \\
\hline \multirow[t]{2}{*}{ Cost } & Health system & Clinical trial data & & & & & $x$ \\
\hline & Societal & Extrapolated & & & & & $x$ \\
\hline
\end{tabular}

CFIR Consolidated Framework for Implementation Research

will finalize the code list and repeat data review using the finalized code structure for coding reliability.

To increase policy impact, we will also conduct a prospective economic evaluation to provide an understanding of the resource implications of the STAR program intervention on post-sepsis health benefits and costs. We will combine primary data and mathematical modeling to examine the costs and cost-effectiveness of STAR over the 90-day study follow up as a measure of benefit from the health system perspective [78]. We will also incorporate evidence-based extrapolations of selected post-sepsis related risk factors, future disease burden, and Markov-type state transition simulation modeling to project cost-effectiveness 12 months after hospital discharge as a measure of benefit from the societal perspective [79-83]. The investigation will collect detailed information on costs associated with the addition of STAR program services (e.g., dedicated program staff - nurse navigators, training) plus the total variable costs of healthcare services received over the 90-day study follow-up period. Quality Adjusted Life Years will be estimated based on utility assessments from published literature [84-86] adjusted for factors at hospital discharge (e.g., ICU days, age, comorbidities), subsequent rehospitalization, and mortality. In both 90-day and 12-month approaches, we will assess the incremental cost effectiveness ratio defined as incremental change in costs divided by incremental change in effectiveness. These data will provide benchmarks to support resource allocation for STAR program scale-up and spread and inform health policy decisions related to post-sepsis care [87].

\section{Data and safety monitoring}

The ENCOMPASS trial is overseen by a Data and Safety Monitoring Board (DSMB) comprising experts in critical care, infectious disease, internal medicine, biostatistics, 
and epidemiology. The DSMB and study investigators agreed not to establish formal guidelines for stopping but are monitoring 90-day all-cause mortality rates and major depressive disorder using the Patient Health Questionnaire-9 [88] as safety endpoints. Due to the low risk associated with study participation, there are no planned interim analyses of the primary outcome.

\section{Discussion}

Healthcare systems urgently need programs to better support sepsis survivors following hospital discharge - a key priority that has been intensified as a result of the coronavirus disease 2019 pandemic and anticipated increasing number of individuals impacted by sepsis [89]. The ENCOMPASS stepped-wedge hybrid type I effectivenessimplementation trial advances knowledge of implementation and delivery of post-sepsis care in multiple ways. First, despite the societal burden and cost associated with sepsis, there is limited evidence to guide management of patients after hospitalization for sepsis. The STAR program is a telehealth nurse navigator-led post-sepsis transition intervention that combines multiple evidence-based components for application in a heterogenous healthcare setting. STAR and its evaluation have been designed with elements to enhance generalizability of results (e.g., diverse study hospitals, expert care recommendations, international guidelines for clinical identification of sepsis patients) and sustainability and scalability to multiple settings locally, regionally, and nationally (e.g., resourceconservative, virtually connected navigators). For example, using telehealth to deliver post-sepsis care elements overcomes the barriers identified in the literature concerning accessibility and reach of clinic-based follow-up care for survivors of critical illness [90]. Further, the telehealth platform extends the reach of nurse navigators across a geographically diverse cohort.

Second, effectiveness studies for complex healthcare problems like this one too often neglect the vital importance of evaluating and adapting implementation practices based on robust implementation science frameworks and constructs. Similarly, there has been no dissemination and implementation research specifically on post-sepsis care in the context of a determinants framework to establish effective processes and their outcomes. Our proposal is innovative in its emphasis on optimization of dissemination and implementation of post-sepsis care in heterogenous healthcare settings, while enhancing traditional implementation frameworks with focused ethnography to obtain a more nuanced understanding of the nurse navigator's role in implementation success. By evaluating the implementation and effectiveness of the STAR intervention across eight diverse hospitals, we will generate knowledge that will immediately be relevant for a broad range of acute care settings and healthcare systems. Additionally, incorporating cost effectiveness analyses will further provide key information to stakeholders to guide future use and scale-up of the STAR program, if shown to be effective and cost-favorable. Thus, the proposed project has the potential to shift the paradigm of how post-sepsis care can be more effectively implemented and disseminate the information widely to improve care and costs for millions of sepsis survivors.

\section{Abbreviations}

AH: Atrium Health; CFIR: Consolidated Framework for Implementation Research; DSMB: Data and Safety Monitoring Board; EDW: Enterprise Data Warehouse; EHR: Electronic Health Record; ENCOMPASS: Engagement and Collaborative Management to Proactively Advance Sepsis Survivorship; PREC IS-2: Pragmatic Explanatory Continuum Indicator Summary 2; RE-AIM: Reach, Effectiveness, Adoption, Implementation, and Maintenance; SOFA: Sequential Organ Failure Assessment; SPIRIT: Standardized Protocol Items:

Recommendations for Interventional Trials; STAR: Sepsis Transition and Recovery

\section{Supplementary Information}

The online version contains supplementary material available at https://doi. org/10.1186/s12913-021-06521-1.

\section{Additional file 1.}

\section{Acknowledgements}

We acknowledge the 1) multidisciplinary team that makes the Sepsis Transition and Recovery program possible, including: Aleta Rios, BSN; Stephanie Murphy, DO; Barb DeSilva, MSN, MHA; Diamond Staton-Williams, $\mathrm{RN}, \mathrm{BSN}, \mathrm{MHA}, \mathrm{ACM}, \mathrm{BC}-\mathrm{NE} ; \mathrm{2}$ ) collaboration of the Atrium Health Acute Care Outcomes Research Network Investigators listed here (in alphabetical order): Ryan Brown, MD; Larry Burke, MD; Shih-Hsiung Chou, PhD; Kyle Cunningham, MD; Susan L. Evans, MD; Scott Furney, MD; Michael Gibbs, MD; Bella Gutnik, MS; Alan Heffner, MD; Timothy Hetherington, MS; Daniel Howard, MD; Marc Kowalkowski, PhD; Scott Lindblom, MD; Andrea McCall; Lewis McCurdy, MD; Andrew McWilliams, MD, MPH; Stephanie Murphy, DO; Alfred Papali, MD; Christopher Polk, MD; Whitney Rossman, MS; Michael Runyon, MD; Mark Russo, MD; Brice Taylor, MD; Stephanie Taylor, MD, MS; 3) clinical sites and hospital leadership participating in the ENCOMPASS trial; and 4) valuable contributions of the Data and Safety Monitoring Board: Melanie Spencer, PhD (Chair); Rita Bakhru, MD; James Horton, MD; Nicholas Pajewski, PhD; and Bennal Perkins, MD.

\section{Authors' contributions}

MK, TE, AM, HT, AR, SM, RB, BG, KO, LM, MD, CB, SC, SH, DA, and ST developed the study design and protocol; $M K$ and ST drafted the manuscript; and MK, TE, AM, HT, AR, SM, RB, BG, KO, LM, MD, CB, SC, SH, DA, and ST critically reviewed, revised, and approved the final manuscript.

\section{Funding}

Research reported in this publication was independently peer-reviewed and supported by National Institute of Nursing Research of the National Institutes of Health under award number R01NR018434. The content is solely the responsibility of the authors and does not necessarily represent the official views of the National Institutes of Health.

Availability of data and materials

Datasets generated during the current study will not be shared, consistent with data governance and policies.

\section{Declarations}

Ethics approval and consent to participate

This study has been approved to be conducted on behalf of all trial sites with a waiver of informed consent by the Advarra Institutional Review Board 
(\#Pro00036873) on June 26, 2019. The trial was retrospectively registered at ClinicalTrials.gov (NCT04495946), first submitted on July 7, 2020 and posted August 3, 2020.

\section{Consent for publication}

Not applicable.

\section{Competing interests}

Dr. Blanchette reports receiving salary from Novo Nordisk. No other disclosures were reported.

\section{Author details}

${ }^{1}$ Center for Outcomes Research and Evaluation, Atrium Health, $1300 \mathrm{Scott}$ Ave, Charlotte, NC 28203, USA. ²Department of Internal Medicine, Atrium Health, Charlotte, USA. ${ }^{3}$ Department of Family Medicine, Atrium Health, Charlotte, USA. ${ }^{4}$ Ambulatory Care Management, Atrium Health, Charlotte, USA. ${ }^{5}$ Division of Infectious Disease, Department of Internal Medicine, Atrium Health, Charlotte, USA. ${ }^{6}$ Academy for Population Health Innovation, University of North Carolina Charlotte \& Mecklenburg County Public Health Department, Charlotte, USA. 'Department of Public Health Sciences, University of North Carolina Charlotte, Charlotte, USA. ${ }^{8}$ Health Economics and Outcomes Research Strategy, Novo Nordisk, Plainsboro Township, USA. ${ }^{9}$ Palliative and Advanced IIlness Research (PAIR) Center, Perelman School of Medicine, University of Pennsylvania, Philadelphia, USA. ${ }^{10}$ Department of Medicine, Perelman School of Medicine, University of Pennsylvania, Philadelphia, USA. ${ }^{11}$ Clinical Research, Investigation, and Systems Modeling of Acute illness (CRISMA) Center, University of Pittsburgh, Pittsburgh, USA. ${ }^{12}$ Department of Critical Care Medicine, University of Pittsburgh, Pittsburgh, USA.

\section{Received: 3 May 2021 Accepted: 12 May 2021}

Published online: 02 June 2021

\section{References}

1. Fleischmann C, Scherag A, Adhikari NK, et al. Assessment of global incidence and mortality of hospital-treated Sepsis. Current estimates and limitations. Am J Respir Crit Care Med. 2016;193(3):259-72. https://doi.org/1 0.1164/rccm.201504-07810C.

2. Rhodes A, Evans LE, Alhazzani W, Levy MM, Antonelli M, Ferrer R, et al. Surviving Sepsis campaign: international guidelines for Management of Sepsis and Septic Shock: 2016. Intensive Care Med. 2017;43(3):304-77. https://doi.org/10.1007/s00134-017-4683-6.

3. Kaukonen KM, Bailey M, Suzuki S, Pilcher D, Bellomo R. Mortality related to severe sepsis and septic shock among critically ill patients in Australia and New Zealand, 2000-2012. JAMA. 2014;311(13):1308-16. https://doi.org/10.1 001/jama.2014.2637

4. Iwashyna TJ, Ely EW, Smith DM, Langa KM. Long-term cognitive impairment and functional disability among survivors of severe sepsis. JAMA. 2010; 304(16):1787-94. https://doi.org/10.1001/jama.2010.1553.

5. Shah FA, Pike F, Alvarez K, Angus D, Newman AB, Lopez O, et al. Bidirectional relationship between cognitive function and pneumonia. Am J Respir Crit Care Med. 2013;188(5):586-92. https://doi.org/10.1164/rccm.2 01212-21540C.

6. Schuler A, Wulf DA, Lu Y, Iwashyna TJ, Escobar GJ, Shah NH, et al. The impact of acute organ dysfunction on long-term survival in Sepsis. Crit Care Med. 2018;46(6):843-9. https://doi.org/10.1097/CCM.0000000000003023.

7. Borges RC, Carvalho CR, Colombo AS, da Silva Borges MP, Soriano FG. Physical activity, muscle strength, and exercise capacity 3 months after severe sepsis and septic shock. Intensive Care Med. 2015;41(8):1433-44. https://doi.org/10.1007/s00134-015-3914-y.

8. Annane D, Sharshar T. Cognitive decline after sepsis. Lancet Respir Med 2015;3(1):61-9. https://doi.org/10.1016/S2213-2600(14)70246-2.

9. Jackson JC, Hopkins RO, Miller RR, Gordon SM, Wheeler AP, Ely EW. Acute respiratory distress syndrome, sepsis, and cognitive decline: a review and case study. South Med J. 2009;102(11):1150-7. https://doi.org/10.1097/SMJ. Ob013e3181b6a592

10. Prescott HC, Langa KM, Iwashyna TJ. Readmission diagnoses after hospitalization for severe sepsis and other acute medical conditions. JAMA. 2015;313(10):1055-7. https://doi.org/10.1001/jama.2015.1410.

11. Mayr FB, Talisa VB, Balakumar V, Chang CH, Fine M, Yende S. Proportion and cost of unplanned 30-day readmissions after Sepsis compared with other medical conditions. JAMA. 2017;317(5):530-1. https://doi.org/10.1001/jama.2 016.20468.

12. Prescott HC, Angus DC. Enhancing recovery from Sepsis: a review. JAMA. 2018;319(1):62-75. https://doi.org/10.1001/jama.2017.17687.

13. Taylor SP, Chou SH, Sierra MF, Shuman TP, McWilliams AD, Taylor BT, et al. Association between adherence to recommended care and outcomes for adult survivors of Sepsis. Ann Am Thorac Soc. 2020;17(1):89-97. https://doi. org/10.1513/AnnalsATS.201907-5140C.

14. Taylor S, Figueroa-Sierra M, Shuman T, et al. Post-sepsis care recommendations are associated with improved patient outcomes but adherence is low [abstract]. Crit Care Med. 2019;47(1):636. https://doi.org/1 0.1097/01.ccm.0000552064.94567.7c.

15. Brownson RC, Allen P, Duggan K, Stamatakis KA, Erwin PC. Fostering moreeffective public health by identifying administrative evidence-based practices: a review of the literature. Am J Prev Med. 2012;43(3):309-19. https://doi.org/10.1016/j.amepre.2012.06.006.

16. Bodenheimer T. Coordinating care--a perilous journey through the health care system. N Engl J Med. 2008;358(10):1064-71. https://doi.org/10.1056/ NEJMhpr0706165.

17. Coleman EA, Berenson RA. Lost in transition: challenges and opportunities for improving the quality of transitional care. Ann Intern Med. 2004;141(7): 533-6. https://doi.org/10.7326/0003-4819-141-7-200410050-00009.

18. Kim CS, Flanders SA. In the clinic. Transitions of care. Ann Intern Med. 2013; 158(5 Pt 1):ITC3-1. https://doi.org/10.7326/0003-4819-158-5-201303050-01003.

19. Tschudy MM, Raphael JL, Nehal US, O'Connor KG, Kowalkowski M, Stille CJ. Barriers to care coordination and medical home implementation. Pediatrics. 2016;138(3):e20153458.

20. Curran GM, Bauer M, Mittman B, Pyne JM, Stetler C. Effectivenessimplementation hybrid designs: combining elements of clinical effectiveness and implementation research to enhance public health impact. Med Care. 2012;50(3):217-26. https://doi.org/10.1097/MLR.0b013e31 82408812

21. Hansen LO, Young RS, Hinami K, Leung A, Williams MV. Interventions to reduce 30-day rehospitalization: a systematic review. Ann Intern Med. 2011; 155(8):520-8. https://doi.org/10.7326/0003-4819-155-8-201110180-00008.

22. Naylor MD, Aiken LH, Kurtzman ET, Olds DM, Hirschman KB. The care span: the importance of transitional care in achieving health reform. Health Aff (Millwood). 2011;30(4):746-54. https://doi.org/10.1377/hlthaff.2011.0041.

23. Barker D, McElduff P, D'Este C, Campbell MJ. Stepped wedge cluster randomised trials: a review of the statistical methodology used and available. BMC Med Res Methodol. 2016;16(1):69. https://doi.org/10.1186/s12 874-016-0176-5.

24. Weijer C, Grimshaw JM, Eccles MP, McRae AD, White A, Brehaut JC, et al. The Ottawa statement on the ethical design and conduct of cluster randomized trials. PLoS Med. 2012;9(11):e1001346. https://doi.org/10.1371/ journal.pmed.1001346.

25. Chan AW, Tetzlaff JM, Altman DG, Laupacis A, Gøtzsche PC, Krleža-Jerić K, et al. SPIRIT 2013 statement: defining standard protocol items for clinical trials. Ann Intern Med. 2013;158(3):200-7. https://doi.org/10.7326/0003-481 9-158-3-201302050-00583.

26. Hemming K, Taljaard M, McKenzie JE, et al. Reporting of stepped wedge cluster randomised trials: extension of the CONSORT 2010 statement with explanation and elaboration. BMJ. 2018:363:k1614.

27. Loudon K, Treweek S, Sullivan F, Donnan P, Thorpe KE, Zwarenstein M. The PRECIS-2 tool: designing trials that are fit for purpose. BMJ. 2015;350(may08 1):h2147. https://doi.org/10.1136/bmj.h2147.

28. Wagner EH. Chronic disease management: what will it take to improve care for chronic illness? Eff Clin Pract. 1998;1(1):2-4.

29. Coleman K, Austin BT, Brach C, Wagner EH. Evidence on the chronic care model in the new millennium. Health Aff (Millwood). 2009;28(1):75-85. https://doi.org/10.1377/hlthaff.28.1.75

30. Parker VA, Lemak $\mathrm{CH}$. Navigating patient navigation: crossing health services research and clinical boundaries. Adv Health Care Manag. 2011;11:149-83. https://doi.org/10.1108/S1474-8231(2011)0000011010.

31. Freeman HP. The history, principles, and future of patient navigation: commentary. Semin Oncol Nurs. 2013;29(2):72-5. https://doi.org/10.1016/j. soncn.2013.02.002

32. Brenner AT, Hoffman R, McWilliams A, Pignone MP, Rhyne RL, Tapp H, et al. Colorectal Cancer screening in vulnerable patients: promoting informed and shared decisions. Am J Prev Med. 2016;51(4):454-62. https://doi.org/10.1016/ j.amepre.2016.03.025. 
33. Shlay JC, Barber B, Mickiewicz T, Maravi M, Drisko J, Estacio R, et al. Reducing cardiovascular disease risk using patient navigators, Denver, Colorado, 2007-2009. Prev Chronic Dis. 2011;8(6):A143.

34. Scott LB, Gravely S, Sexton TR, Brzostek S, Brown DL. Examining the effect of a patient navigation intervention on outpatient cardiac rehabilitation awareness and enrollment. J Cardiopulm Rehabil Prev. 2013;33(5):281-91. https://doi.org/10.1097/HCR.0b013e3182972dd6.

35. Damschroder LJ, Aron DC, Keith RE, Kirsh SR, Alexander JA, Lowery JC. Fostering implementation of health services research findings into practice: a consolidated framework for advancing implementation science. Implement Sci. 2009:4(1):50. https://doi.org/10.1186/1748-5908-4-50.

36. Kirk MA, Kelley C, Yankey N, Birken SA, Abadie B, Damschroder L. A systematic review of the use of the consolidated framework for implementation research. Implement Sci. 2016;11:72.

37. HCUPnet, Healthcare Cost and Utilization Project. Agency for Healthcare Research and Quality, Rockville, MD. https://hcupnet.ahrq.gov/. For more information about HCUP data see http://www.hcup-us.ahrq.gov/.

38. Rhee C, Dantes R, Epstein L, Murphy DJ, Seymour CW, Iwashyna TJ, et al. Incidence and trends of Sepsis in US hospitals using clinical vs claims data, 2009-2014. JAMA. 2017;318(13):1241-9. https:/doi.org/10.1001/jama.2017.13836.

39. Mostel Z, Perl A, Marck M, Mehdi SF, Lowell B, Bathija S, et al. Post-sepsis syndrome - an evolving entity that afflicts survivors of sepsis. Mol Med. 2019;26(1):6. https://doi.org/10.1186/s10020-019-0132-z

40. Fernando SM, Tran A, Taljaard M, Cheng W, Rochwerg B, Seely AJE, et al. Prognostic accuracy of the quick sequential organ failure assessment for mortality in patients with suspected infection: a systematic review and meta-analysis. Ann Intern Med. 2018;168(4):266-75. https://doi.org/10.7326/ M17-2820.

41. Williams JM, Greenslade JH, McKenzie JV, Chu K, Brown AFT, Lipman J. Systemic inflammatory response syndrome, quick sequential organ function assessment, and organ dysfunction: insights from a prospective database of ED patients with infection. Chest. 2017;151(3):586-96. https://doi.org/10.101 6/j.chest.2016.10.057

42. Singer M, Deutschman CS, Seymour CW, Shankar-Hari M, Annane D, Bauer $M$, et al. The third international consensus definitions for Sepsis and septic shock (Sepsis-3). JAMA. 2016;315(8):801-10. https://doi.org/10.1001/jama.201 6.0287.

43. Seymour CW, Liu VX, Iwashyna TJ, Brunkhorst FM, Rea TD, Scherag A, et al. Assessment of clinical criteria for Sepsis: for the third international consensus definitions for Sepsis and septic shock (Sepsis-3). JAMA. 2016; 315(8):762-74. https://doi.org/10.1001/jama.2016.0288.

44. Raith EP, Udy AA, Bailey M, McGloughlin S, Maclsaac C, Bellomo R, et al. Prognostic accuracy of the SOFA score, SIRS criteria, and qSOFA score for in-hospital mortality among adults with suspected infection admitted to the intensive care unit. JAMA. 2017;317(3):290-300. https://doi.org/10.1001/ja ma.2016.20328.

45. de Grooth HJ, Geenen IL, Girbes AR, Vincent JL, Parienti JJ, Oudemans-van Straaten HM. SOFA and mortality endpoints in randomized controlled trials: a systematic review and meta-regression analysis. Crit Care. 2017;21(1):38. https://doi.org/10.1186/s13054-017-1609-1.

46. Shankar-Hari M, Saha R, Wilson J, Prescott HC, Harrison D, Rowan K, et al. Rate and risk factors for rehospitalisation in sepsis survivors: systematic review and meta-analysis. Intensive Care Med. 2020;46(4):619-36. https://doi. org/10.1007/s00134-019-05908-3.

47. Prescott HC, Iwashyna TJ, Blackwood B, et al. Understanding and enhancing sepsis survivorship: priorities for research and practice. Am J Respir Crit Care Med. 2019;200:972

48. Prescott HC, Costa DK. Improving long-term outcomes after Sepsis. Crit Care Clin. 2018;34(1):175-88. https://doi.org/10.1016/j.ccc.2017.08.013.

49. Kowalkowski M, Chou SH, McWilliams A, et al. Structured, proactive care coordination versus usual care for improving morbidity during post-acute care transitions for Sepsis (IMPACTS): a pragmatic, randomized controlled trial. Trials. 2019;20(1):660. https://doi.org/10.1186/s13063-019-3792-7.

50. McWilliams A, Roberge J, Anderson WE, Moore CG, Rossman W, Murphy S, et al. Aiming to improve readmissions through InteGrated hospital transitions (AIRTIGHT): a pragmatic randomized controlled trial. J Gen Intern Med. 2019;34(1):58-64. https://doi.org/10.1007/s11606-018-4617-1.

51. Harris PA, Taylor R, Thielke R, Payne J, Gonzalez N, Conde JG. Research electronic data capture (REDCap)--a metadata-driven methodology and workflow process for providing translational research informatics support. J Biomed Inform. 2009;42(2):377-81. https://doi.org/10.1016/j.jbi.2008.08.010.
52. Gaudry S, Messika J, Ricard JD, Guillo S, Pasquet B, Dubief E, et al. Patientimportant outcomes in randomized controlled trials in critically ill patients: a systematic review. Ann Intensive Care. 2017;7(1):28. https://doi.org/10.1186/ s13613-017-0243-z.

53. Liu V, Escobar GJ, Greene JD, Soule J, Whippy A, Angus DC, et al. Hospital deaths in patients with sepsis from 2 independent cohorts. JAMA. 2014; 312(1):90-2. https://doi.org/10.1001/jama.2014.5804.

54. Berenson RA, Paulus RA, Kalman NS. Medicare's readmissions-reduction program--a positive alternative. N Engl J Med. 2012;366(15):1364-6. https:// doi.org/10.1056/NEJMp1201268.

55. Zuckerman RB, Sheingold SH, Orav EJ, Ruhter J, Epstein AM. Readmissions, observation, and the hospital readmissions reduction program. N Engl J Med. 2016;374(16):1543-51. https://doi.org/10.1056/NEJMsa1513024.

56. Gupta A, Allen LA, Bhatt DL, Cox M, DeVore AD, Heidenreich PA, et al. Association of the Hospital Readmissions Reduction Program Implementation with Readmission and Mortality Outcomes in heart failure. JAMA Cardiol. 2018:3(1):44-53. https://doi.org/10.1001/jamacardio.2017.4265.

57. Quinn J, Kramer N, McDermott D. Validation of the social security death index (SSDI): an important readily-available outcomes database for researchers. West J Emerg Med. 2008;9(1):6-8.

58. Groff AC, Colla CH, Lee TH. Days spent at home - a patient-centered goal and outcome. N Engl J Med. 2016;375(17):1610-2. https://doi.org/10.1056/ NEJMp1607206.

59. Dinglas VD, Faraone LN, Needham DM. Understanding patient-important outcomes after critical illness: a synthesis of recent qualitative, empirical, and consensus-related studies. Curr Opin Crit Care. 2018;24(5):401-9. https:// doi.org/10.1097/MCC.0000000000000533.

60. Harhay MO, Casey JD, Clement M, Collins SP, Gayat É, Gong MN, et al. Contemporary strategies to improve clinical trial design for critical care research: insights from the first critical care clinical Trialists workshop. Intensive Care Med. 2020;46(5):930-42. https://doi.org/10.1007/s00134-02005934-6.

61. Rudd KE, Johnson SC, Agesa KM, Shackelford KA, Tsoi D, Kievlan DR, et al. Global, regional, and national sepsis incidence and mortality, 1990-2017: analysis for the global burden of disease study. Lancet. 2020;395(10219): 200-11. https://doi.org/10.1016/S0140-6736(19)32989-7.

62. Agana DFG, Striley CW, Cook RL, Cruz-Almeida Y, Carek PJ, Salemi JL. A novel approach to characterizing readmission patterns following hospitalization for ambulatory care-sensitive conditions. J Gen Intern Med. 2020;35(4):1060-8. https://doi.org/10.1007/s11606-020-05643-2.

63. Hussey MA, Hughes JP. Design and analysis of stepped wedge cluster randomized trials. Contemp Clin Trials. 2007;28(2):182-91. https://doi.org/1 0.1016/j.cct.2006.05.007.

64. Baio G, Copas A, Ambler G, Hargreaves J, Beard E, Omar RZ. Sample size calculation for a stepped wedge trial. Trials. 2015;16(1):354. https://doi.org/1 0.1186/s13063-015-0840-9

65. VanderWeele TJ. On the distinction between interaction and effect modification. Epidemiology. 2009;20(6):863-71. https://doi.org/10.1097/EDE. Ob013e3181ba333c

66. Corraini P, Olsen M, Pedersen L, Dekkers OM, Vandenbroucke JP. Effect modification, interaction and mediation: an overview of theoretical insights for clinical investigators. Clin Epidemiol. 2017;9:331-8. https://doi.org/10.214 7/CLEP.S129728.

67. Knol MJ, VanderWeele TJ. Recommendations for presenting analyses of effect modification and interaction. Int J Epidemiol. 2012;41(2):514-20. https://doi.org/10.1093/ije/dyr218.

68. Fergusson D, Aaron SD, Guyatt G, Hebert P. Post-randomisation exclusions: the intention to treat principle and excluding patients from analysis. BMJ. 2002;325(7365):652-4. https://doi.org/10.1136/bmj.325.7365.652.

69. Little RJ. Pattern-mixture models for multivariate incomplete data. J Am Stat Assoc. 1993;88:125-34

70. Guo W, Ratcliffe SJ, Have TT. A random pattern-mixture model for longitudinal data with dropouts. J Am Stat Assoc. 2004;99(468):929-37. https://doi.org/10.1198/016214504000000674.

71. Prescott HC, Osterholzer JJ, Langa KM, Angus DC, Iwashyna TJ. Late mortality after sepsis: propensity matched cohort study. BMJ. 2016:353: i2375.

72. Rhee C, Jones TM, Hamad Y, Pande A, Varon J, O'Brien C, et al. Prevalence, underlying causes, and preventability of Sepsis-associated mortality in US acute care hospitals. JAMA Netw Open. 2019;2(2):e187571. https://doi.org/1 0.1001/jamanetworkopen.2018.7571. 
73. Hooper R, Teerenstra S, de Hoop E, Eldridge S. Sample size calculation for stepped wedge and other longitudinal cluster randomised trials. Stat Med. 2016;35(26):4718-28. https://doi.org/10.1002/sim.7028.

74. Glasgow RE, Vogt TM, Boles SM. Evaluating the public health impact of health promotion interventions: the RE-AIM framework. Am J Public Health. 1999;89(9):1322-7. https://doi.org/10.2105/AJPH.89.9.1322.

75. Salzmann-Erikson M. Using focused ethnography to explore and describe the process of nurses' shift reports in a psychiatric intensive care unit. I Clin Nurs. 2018;27(15-16):3104-14. https://doi.org/10.1111/jocn.14502.

76. Cruz EV, Higginbottom G. The use of focused ethnography in nursing research. Nurse Res. 2013;20(4):36-43. https://doi.org/10.7748/nr2013.03.2 0.4.36.e305.

77. Bradley EH, Curry LA, Devers KJ. Qualitative data analysis for health services research: developing taxonomy, themes, and theory. Health Serv Res. 2007; 42(4):1758-72. https://doi.org/10.1111/j.1475-6773.2006.00684.x.

78. Ramsey SD, Willke RJ, Glick H, Reed SD, Augustovski F, Jonsson B, et al. Cost-effectiveness analysis alongside clinical trials II-an ISPOR good research practices task force report. Value Health. 2015;18(2):161-72. https://doi.org/1 0.1016/j.jval.2015.02.001.

79. Drabinski A, Williams G, Formica C. Observational evaluation of health state utilities among a cohort of sepsis patients. Value Health. 2001;4(2):128-9.

80. Lee H, Doig CJ, Ghali WA, Donaldson C, Johnson D, Manns B. Detailed cost analysis of care for survivors of severe sepsis. Crit Care Med. 2004;32(4):981-5. https://doi.org/10.1097/01.CCM.0000120053.98734.2C.

81. Karlsson S, Ruokonen E, Varpula T, Ala-Kokko TI, Pettila V, Finnsepsis SG. Long-term outcome and quality-adjusted life years after severe sepsis. Crit Care Med. 2009;37(4):1268-74. https://doi.org/10.1097/CCM.0b013e3181 $9 \mathrm{c} 13 \mathrm{ac}$.

82. Iwashyna TJ. Trajectories of recovery and dysfunction after acute illness, with implications for clinical trial design. Am J Respir Crit Care Med. 2012; 186(4):302-4. https://doi.org/10.1164/rccm.201206-1138ED.

83. Lone NI, Seretny M, Wild SH, Rowan KM, Murray GD, Walsh TS. Surviving intensive care: a systematic review of healthcare resource use after hospital discharge*. Crit Care Med. 2013;41(8):1832-43. https://doi.org/10.1097/CCM. 0b013e31828a409c.

84. Busse LW, Nicholson G, Nordyke RJ, Lee CH, Zeng F, Albertson TE. Angiotensin II for the treatment of distributive shock in the intensive care unit: a US cost-effectiveness analysis. Int J Technol Assess Health Care. 2020; 36(2):145-51. https://doi.org/10.1017/\$0266462320000082.

85. Linder A, Guh D, Boyd JH, Walley KR, Anis AH, Russell JA. Long-term (10year) mortality of younger previously healthy patients with severe sepsis/ septic shock is worse than that of patients with nonseptic critical illness and of the general population. Crit Care Med. 2014;42(10):2211-8. https://doi. org/10.1097/CCM.0000000000000503.

86. Shapiro NI, Howell MD, Talmor D, Donnino M, Ngo L, Bates DW. Mortality in emergency department Sepsis (MEDS) score predicts 1-year mortality. Crit Care Med. 2007;35(1):192-8. https://doi.org/10.1097/01.CCM.0000251508.12 555.3E.

87. Cairns J. Using cost-effectiveness evidence to inform decisions as to which health services to provide. Health Syst Reform. 2016;2(1):32-8. https://doi. org/10.1080/23288604.2015.1124172.

88. Kroenke K, Spitzer RL, Williams JB. The PHQ-9: validity of a brief depression severity measure. J Gen Intern Med. 2001;16(9):606-13. https://doi.org/10.1 046/j.1525-1497.2001.016009606.x.

89. Prescott HC, Girard TD. Recovery from severe COVID-19: leveraging the lessons of survival from Sepsis. JAMA. 2020;324(8):739-40. https://doi.org/1 0.1001/jama.2020.14103.

90. Haines K, McPeake J, Hibbert E, Boehm LM, Aparanji K, Bakhru RN, et al. Enablers and barriers to implementing ICU follow-up clinics and peer support groups following critical illness: the thrive Collaboratives. Crit Care Med. 2019;47(9):1194-200. https://doi.org/10.1097/CCM.0000000000003818

\section{Publisher's Note}

Springer Nature remains neutral with regard to jurisdictional claims in published maps and institutional affiliations.

Ready to submit your research? Choose BMC and benefit from:

- fast, convenient online submission

- thorough peer review by experienced researchers in your field

- rapid publication on acceptance

- support for research data, including large and complex data types

- gold Open Access which fosters wider collaboration and increased citations

- maximum visibility for your research: over $100 \mathrm{M}$ website views per year

At BMC, research is always in progress.

Learn more biomedcentral.com/submissions 